\title{
Correspondence
}

\section{A model for managing violence in acute adult admission wards}

A retrospective survey of contemporaneous electronic case records in a male psychiatric intensive care unit (PICU) in central London was carried out for 2012. The notes were scrutinised for records of serious violence where there was threat to life or limb that resulted in patients being given rapid tranquillisation and seclusion. The survey revealed that of 72 admitted individuals, 58\% were responsible for this degree of behaviour. Most incidents (67\%) were perpetrated in multiples by slightly fewer than $25 \%$ of all those who were admitted. This suggests an average of 3 serious incidents per patient.

In a meta-analysis on in-patient aggression, ${ }^{1}$ a literature review shows that the estimated percentage of aggression on acute admission wards is extremely variable, with figures quoted from 8 to $44 \%$. A third of in-patients have experienced violent or threatening behaviour, with higher figures for staff $41 \%$ of clinical staff and almost $80 \%$ of nursing staff working in in-patient units have experienced aggressive behaviour. It is important therefore to understand the strength of association between risk factors for in-patient aggression and the extent to which these disruptive and distressing events can be predicted and prevented.

In the present retrospective survey, it was clear from the data that the incidence of violence decreased consistently week on week; $45 \%$ of all behaviours $(n=80)$ requiring emergency nursing intervention occurred in the first week of all admissions. This reduced to $15 \%$ by the second week and $7.5 \%$ by the third week, however, by week 8 there was a rise to $13 \%$. This is an interesting observation which may indicate the point at which PICU becomes counterproductive. Department of Health guidelines for PICU admission recommend that admission should not ordinarily exceed 8 weeks. $^{2}$

The observation that the first week represents the highest risk period of an admission fits in well with previous data. This high-risk period could be an opportunity to monitor imminent behaviours through routine enhanced nursing observations, allowing a proactive rather than reactive response style bearing the brunt of staff/patient interactions., ${ }^{3,4}$ The observations of week-on-week reduction in serious violence could be explored further with a case-control study. Although resource intensive, ultimately any procedure that is likely to reduce violence to staff and patients is worth pursuing.

1 Dack C, Ross J, Papadopulous C, Stewart D, Bowers L. A review and meta-analysis of the patient factors associated with psychiatric in-patient aggression. Acta Psychiatr Scand 2013; 127: 255-68.

2 Department of Health. Mental Health Policy Implementation Guide: National Minimal Standards for General Adult Services in Psychiatric Intensive Care Units (PICU) and Low Secure Environments. Department of Health, 2002.

3 Papadopolous C, Ross J, Stewart D, Dack C, James K, Bowers L. The antecedents of violence and aggression within psychiatric in-patient settings. Acta Psychiatr Scand 2012; 125: 425-39.
4 Bowers L, Gournay K, Duffy D. Suicide and self-harm in inpatient psychiatric units: a national survey of observation policies. J Adv Nurs 2000; 32: 437-44.

Reuven M. Magnes, consultant psychiatrist, Southend on Sea, UK, email: thegroov@doctors.org.uk

doi: 10.1192/pb.38.3.137

\section{Neuroimaging in dementia: how best to use the guidelines?}

Kuruvilla et al completed an audit cycle on neuroimaging practice after national and European guidance was adapted to local resource availability. The audit showed an improvement in the number of patients who have had at least one form of neuroimaging performed from 68 to $76 \%$, and although this was not statistically significant, it seems to suggest a general improvement in the service provided, as reflected also in the improved documentation of the reason for not requesting neuroimaging and in having no significant impact on waiting times. Improvement in the service may also be reflected in a patient and relative satisfaction survey that could be carried out.

In a similar study (details available from the author on request), I audited the practice of a memory clinic in Southport, Merseyside, against 2006 National Institute for Health and Care Excellence (NICE) guidance on dementia, ${ }^{2}$ which stated that 'structural imaging should be used in the assessment of people with suspected dementia' and that magnetic resonance imaging (MRI) 'is the preferred modality [...] although computed tomography (CT) scanning could be used'. The audit included 75 patients and showed that 56 (75\%) had at least one neuroimaging procedure performed: 53 (95\%) of these had CT scans and only 1 patient had an MRI scan. My audit revealed a similar problem with documentation of reasons for not scanning patients, with $31 \%$ of patients who were not scanned lacking such documentation compared with $50 \%$ in Kuruvilla et al's initial audit. In my study a re-audit was not carried out.

An additional aim of my study was to look at whether the diagnosis of dementia subtype, provisionally made based on clinical interview and using scales such as MMSE and ACE-R, was changed following neuroimaging. This revealed that the diagnosis was changed following a scan in $45 \%$ of cases, mostly from Alzheimer's or vascular dementia into a mixedtype dementia. It also showed that no provisional diagnosis was documented in $38 \%$ of case notes reviewed, suggesting that clinicians were perhaps uncomfortable about making a diagnosis before a scan was performed.

Bearing in mind that NICE guidelines are driven partly by cost-effectiveness, studies such as Kuruvilla et al's provide good support for the usefulness of adapting these guidelines to the local availability of resources, which results in better care for patients with dementia.

1 Kuruvilla T, Zheng R, Soden B, Greef S, Lyburn I. Neuroimaging in a memory assessment service: a completed audit cycle. Psychiatr Bull 2014; 38: 24-8. 
2 National Institute for Health and Clinical Excellence. Dementia: Supporting People with Dementia and Their Carers in Health and Social Care (Clinical Guidelines, CG42). NICE, 2006.

Mustafa Alachkar, SpR in psychiatry, Manchester Mental Health and Social Care NHS Trust, Manchester, UK, email: mustafa.alachkar@mhsc.nhs.uk

doi: 10.1192/pb.38.3.137a

\section{Exposure to acute child psychiatry presentations for core psychiatrists}

We are writing to draw attention to the lack of clarity provided by the Royal College of Psychiatrists regarding the role of the core trainee psychiatrist in assessing child and adolescent psychiatry patients out of hours. We believe it is important this issue is addressed as it confers broad implications for training, recruitment and service delivery. Crises of paediatric mental health tend to present out of hours. Ireland's 4th annual child and adolescent mental health service report details 'striking patterns in the number of [self-harm] presentations seen': 51\% of presentations were in the 8 -hour period of $7 \mathrm{pm}$ to $3 \mathrm{am}{ }^{1}$ This finding appears typical for paediatric psychiatry liaison services around the UK.

It is well known that in some trusts core trainees are excluded from child and adolescent mental health services (CAMHS)-led out-of-hours care pathways. This situation seems particularly unsatisfactory given that placements in developmental psychiatry are no longer obligatory. By failing to adequately furnish our future adult psychiatrists with skills in child and adolescent mental health, we are reinforcing a culture whereby young people are potentially falling through the care gap between CAMHS and adult mental health services. ${ }^{2,3}$ Indeed, this very issue is highlighted in a joint paper from the inter-faculty group of the child and adolescent psychiatry and the general and community psychiatry faculties which presents recommendations for the provision of psychiatric services to adolescents and young adults. ${ }^{4}$ Furthermore, by restricting the level of exposure to child psychiatry, we are doing little to encourage core trainees to perceive the specialty as a future career option.

As well as having an impact on the quality of training, the issue has far-reaching implications for patient care. The current lack of clarity fosters an atmosphere of uncertainty as situations arise where no one knows who holds responsibility to clerk a young person on arrival, thereby leading to potential delays in the patient being seen. Emergency department delays are a source of great concern to acute care trusts and create negative attitudes to psychiatric services in general. If we cannot manage to work in a safe and effective way, we are further contributing to the hostility not only towards our specialty but also to our patients, who are at their most vulnerable.

It is therefore our view that there should be an explicit expectation for core trainees to have exposure to the full range of acute psychiatric presentations, including child and adolescent patients, out of hours. It is of course essential that this experience would be supported by robust and accessible supervision structures in the form of a second on-call specialty trainee or consultant child psychiatrist. Although we recognise that the College is unable to tell trusts how to deliver their outof-hours services, it would be helpful if the core psychiatry curriculum contained more robust guidance as to the role of the core trainee in assessing child and adolescent psychiatry cases out of hours. Such a move would help to create clarity as well as holding local education providers to account.

\section{Declaration of interest}

R.C. sits on the College's Emergency Care Taskforce, which is currently considering the value of out-of-hours training.

1 Health Service Executive. Fourth Annual Child \& Adolescent Mental Health Service Report 2011-2012. HSE, 2012 (http://www.hse.ie/eng/services/ Publications/services/Mentalhealth/camhs20112012annualreport.pdf).

2 Singh SP. Transition of care from child to adult mental health services; the great divide. Curr Opin Psychiatry 2009; 22: 386-90.

3 Singh SP, Paul M, Ford T, Kramer T, Weaver T. Transitions of care from child and adolescent mental health services to adult mental health services (TRACK study): a study of protocols in Greater London. BMC Health Serv Res 2008; 8: 1-7.

4 Lamb C, Hill D, Kelvin R, Van Beinum M. Working at the CAMHS/Adult Interface: Good Practice Guidance for the Provision of Psychiatric Services to Adolescents/Young Adults. A Joint Paper from the Interfaculty Working Group of the Child and Adolescent Faculty and the General and Community Faculty of the Royal College of Psychiatrists, May 2008. Royal College of Psychiatrists, 2008.

Amanda K. Shine is ST6 in child and adolescent psychiatry and Rory Conn is ST4 in child and adolescent psychiatry, both at Tavistock and Portman NHS Foundation Trust, London, UK, email: amanda.shine@nhs.net, and Zaib Davids is consultant child and adolescent psychiatrist, Child and Adolescent Psychiatry Liaison Team, University College London Hospital. doi: $10.1192 / p b .38 .3 .138$

\section{Psychiatry for medical students: need for a more holistic approach to teaching?}

We are two medical students who wish to offer a perspective on undergraduate education and psychiatry.

During our student placement, we attended the old age psychiatry module at the Northern Deanery MRCPsych programme focusing on dementia and ethics. This was aimed at trainees and not specifically medical students but we were surprised to find that this was not above our level of knowledge. This prompted discussion of undergraduate psychiatry training more broadly, which we felt focused too heavily on the diagnosis of mental illness and less so on the holistic approach to the patient and their presentation as covered by the MRCPsych course. From our experience of undergraduate psychiatry we feel that the assessment by means of a logbook of conditions encourages students to find patients with a certain diagnosis, and in doing so overlooks the true essence of psychiatry. To our mind this incorporates the ability to consider all aspects of a patient's life and formulating these, while demonstrating compassion for another person at a time of most need.

Through choosing a 6-week placement in old age psychiatry we have been able to explore the specialty more thoroughly and broadly than facilitated within the standard undergraduate programme, and we have realised how little of psychiatry we have been exposed to as undergraduates. We have become more aware of the importance of considering the patient's personal and social circumstances alongside their diagnosis, and how these can influence each other. Specifically, the importance of a sound ethical approach to practice has been highlighted through the higher-level teaching we 
experienced, where the Mental Capacity Act was discussed in detail.

We believe that it would benefit undergraduates to experience a more realistic and rounded placement in psychiatry and truly consider the social implications of mental illness. As it currently stands, undergraduate education in psychiatry is oversimplified to focus on diagnosis and does not acknowledge the capabilities of medical students to adopt a holistic approach. An opportunity to consider all aspects of a psychiatrist's role may encourage more students to consider a career in this field.

Kristina Rodney and Laura Wilkinson are both ST4 medical students, Newcastle University, Newcastle, UK, email: k.rodney@newcastle.ac.uk. doi: $10.1192 / \mathrm{pb} \cdot 38.3 .138 \mathrm{a}$

\section{Factors associated with the use of community treatment orders}

In his article Curtis ${ }^{1}$ highlights one of the limitations of the OCTET study, ${ }^{2}$ in that patients selected for randomisation may not have been suitable for community treatment order (CTO) placement in the first place. In his conclusions he suggests there may be a small subgroup of patients for whom CTOs are enormously beneficial. Perhaps clinicians need more clarity of the characteristics of the 'revolving door' patient better to assess suitability for supervised community treatment.

Most clinicians will have a personal construct of the epidemiological and clinical characteristics of revolving door patients, although this may not be explicitly defined. There is no consistency in the literature as to the definition of revolving door, and previous research in the UK has shown that predictors of readmission are varied and not consistently replicated across studies. Research carried out when the practice of 'long leash' Section 17 leave was widespread showed that those placed on extended leave had a history of more frequent compulsory admissions, increased recent dangerousness to others, and decreased adherence to their out-patient follow-up prior to admission. ${ }^{3}$

A case-control study was conducted at Leeds Partnership NHS Foundation Trust in 2010, and approved by the local research and development department as a service evaluation. The aim was to compare characteristics of patients placed on CTOs and those discharged from Section 3, to elicit which factors were associated with CTO placement. All patients placed on a CTO between November 2008 and February 2010 were included as cases, and controls were randomly selected from patients who had been detained under Section 3 of the Mental Health Act, but whose Section was rescinded within the same week that the CTO was commenced. A ratio of two controls for each case increased the power of the study. This amounted to 56 cases and 112 controls. Characteristics chosen for analysis were those which previous research had suggested may be of importance and where collection was feasible. The characteristics of the patients placed on CTOs were broadly similar to those recruited into OCTET.

Analysing variables individually, patients on CTOs were significantly more likely $(P<0.05)$ to be single, have a principal diagnosis of schizophrenia, a history of violence, a higher number of previous admissions, a history of criminal conviction and a higher number of convictions within the past year.
On logistic regression analysis, patients on CTOs were significantly more likely to have a principal diagnosis of schizophrenia and a higher number of previous admissions.

There remains the outstanding question of who belongs to the elusive group of patients for which CTOs are effective, if indeed this group exists. This study provides insight into the demographic and historical factors that are influencing clinicians' decisions to implement CTOs. There is no proof so far that CTOs are effective in their aims. Perhaps we need to look again at who the truly 'revolving door' patients are and take this objective evidence into consideration at the point of deciding whether to initiate supervised community treatment.

1 Curtis D. OCTET does not demonstrate a lack of effectiveness for community treatment orders. Psychiatr Bull 2014; 38: 36-9.

2 Burns T, Racks J, Molodynski A, Dawson J, Yeeles K, Vazquez-Montes $M$, et al. Community treatment orders for patients with psychosis (OCTET): a randomised controlled trial. Lancet 2013; 381: 1627-33.

3 Sensky T, Hughes T, Hirsch S. Compulsory psychiatric treatment in the community. 1. A controlled study of compulsory community treatment with extended leave under the Mental Health Act: special characteristics of patients treated and the impact of treatment. $\mathrm{Br}$ Psychiatry 1991; 158: 792-9.

Rachel J. McKie, ST5 Psychiatrist, Leeds and York Partnership NHS Foundation Trust, UK, email: rmckie@nhs.net

doi: 10.1192/pb.38.3.139

\section{Misunderstanding recall}

Smith et al should be congratulated for their investigation into the use of the additional conditions that are sometimes included in community treatment orders (CTOs). The Reference Guide to the Mental Health Act 1983 (15.16-15.19) and the Mental Health Act Code of Practice (25.29-25.35) describe the nature of these conditions and how they relate to the recall of patients. Although patients do not have to consent formally to CTOs, or the conditions, in practice they will need to attempt to cooperate with them. However, these additional conditions are not directly enforceable. The Reference Guide (15.30) sets out the criteria the responsible clinician must use when considering recall. These criteria do not refer to additional conditions, and there is no power of recall if a patient on a CTO fails to comply with them. I agree with Smith et al when they claim that many patients on CTOs wrongly believe that if they are unable to adhere to additional conditions they will inevitably be recalled to hospital, and that the prevalence of this misunderstanding is inconsistent with the principles set out in chapter 1 of the Mental Health Act Code of Practice. One of the roles of independent mental health advocates is helping patients obtain information about, and understand their rights under, the Mental Health Act 1983. In my opinion this is an issue that they should prioritise, as should all those who monitor the use of the Act. As Smith et al point out, these circumstances raise serious legal and ethical issues.

1 Smith M, Branton T, Cardno A. Is the bark worse than the bite? Additional conditions used within community treatment orders. Psychiatr Bull 2014; 38: 9-12.

Keith E. Dudleston, consultant psychiatrist (retired), Ivybridge, UK, email: dudleston@btinternet.com

doi: 10.1192/pb.38.3.139a 


\section{Options in managing alternatives to hyoscine in clozapine-induced hypersalivation: a survey of secure services consultants}

Clozapine-induced hypersalivation is socially embarrassing and potentially life threatening. It can lead to poor medication adherence, which is of concern for patients in secure settings.

Hyoscine hydrobromide is widely used as a first-line treatment, despite little available evidence. ${ }^{1}$ Alternatives are limited, but 19 different agents are listed in the Maudsley Prescribing Guidelines, ${ }^{1}$ including antipsychotics, antidepressants and other drugs with antimuscarinic properties. There are few meaningful trials. Within the north-west of England, obtaining hyoscine has been difficult at times due to supply shortages and so alternatives have been sought.

Partnerships in Care have over 50 consultant psychiatrists nationwide caring for over 1000 in-patients, mostly within secure conditions, with a fair proportion prescribed clozapine. To examine prescribing alternatives to hyoscine, all consultants with clinical responsibilities were contacted regarding their prescribing practices and experiences. Responses were sent back in the form of a patient non-identifiable response via email.

Just under $50 \%$ of consultants replied $(n=23)$. In the absence of hyoscine hydrobromide, there was overall little confidence in alternatives, but clinicians tended to advocate one or two. Atropine, either sublingually or via eye drops was relatively popular and the 8 clinicians that supported its use had some confidence in it. Four clinicians each supported the use of amitriptyline, pirenzepine and trihexyphenidyl. All the medication recommendations received were in the latest Maudsley Prescribing Guidelines in Psychiatry, except for procyclidine. Most options consisted of drugs with antimuscarinic properties such as pirenzepine and trihexyphenidyl. Dose reduction of clozapine was recommended by 1 consultant. The author and another two consultants have had some success with glycopyrrolate syrup, but this is a very expensive option.

Clozapine-induced hypersalivation is a condition potentially difficult to manage. The wide range of options and lack of evidence does not support clinicians in their attempts to continue treatment. In circumstances where patients do not respond to hyoscine, the most popular choice was sublingual atropine. National guidance and further trials are required. The shortage of hyoscine raises legal and ethical questions for patients subject to certification by second-opinion doctors and whether clinicians are likely to request further certification for alternative classes of drugs for hypersalivation.

1 Taylor D, Paton C, Kapur S (eds) The Maudsley Prescribing Guidelines in Psychiatry, 11th Edition. Wiley-Blackwell, 2012.

Yasir Kasmi, consultant forensic psychiatrist, Partnerships in Care, Warrington, UK, email: yasir.kasmi@partnershipsincare.co.uk

doi: $10.1192 / \mathrm{pb} .38 .3 .140$

\section{Cardiovascular monitoring in patients prescribed clozapine}

Wilson et al highlight the ongoing issue of poor physical health monitoring in patients prescribed clozapine. We recently presented a survey which investigated standards of physical health monitoring in adult patients $(n=98)$ prescribed clozapine against standards set out by Maudsley Guidelines in which we found similarly high rates (53\%) of clozapine augmentation and antipsychotic polypharmacy (details available from the authors on request). Moreover, cardiovascular monitoring was poor with only $30 \%$ of patients having had a baseline electrocardiogram prior to initiation of clozapine. Similarly, only $28 \%$ had yearly electrocardiogram monitoring performed once clozapine therapy had been established. Of those patients established on clozapine therapy, 34\% were found to have asymptomatic sinus tachycardia, which was more commonly seen in patients prescribed additional antipsychotic medication than those prescribed clozapine alone $(P<0.001)$. Clinical actions in response to asymptomatic sinus tachycardia varied enormously, with only $12 \%$ of cases having been discussed with local cardiology services.

These findings are of great concern when one considers that clozapine is associated with potentially life-threatening adverse cardiovascular conditions such as myocarditis and cardiomyopathy. ${ }^{2}$ While tachycardia is commonly seen during the early stages of clozapine treatment, occurring in up to $50 \%$ of patients, sustained tachycardia, defined as a heart rate $>100$ bpm for more than 6 months, can precipitate cardiomyopathy and appears to be an independent risk factor for sudden cardiac death. ${ }^{3}$ Reducing clozapine dose and the use of ratelimiting drugs such as beta-blockers have been suggested as potential solutions to this problem, ${ }^{4}$ although these options may not always be clinically appropriate and there appears to be a broad range of approaches in dealing with this.

In response to these findings we have introduced a system whereby initiation of clozapine therapy and its continued prescription by our pharmacy department is contingent on evidence of baseline and continued cardiovascular monitoring. We have also developed a shared care pathway with our local cardiology department ensuring that cardiac monitoring is optimised in this vulnerable patient group and that management of sustained tachycardia is jointly managed by both psychiatric and cardiology services. Information on this shared care pathway is available from the corresponding author.

1 Wilson S, Hamilton R, Callender J, MacManus A, Howitt S, Okpo B. Clozapine antipsychotic polypharmacy: audit of use and patient monitoring. Psychiatrist 2013; 37: 322-5.

2 Kilian JG, Kerr K, Lawrence C, Celermajer DS. Myocarditis and cardiomyopathy associated with clozapine. Lancet 1999; 354: $1841-5$.

3 Shinbane JS, Wood MA, Jensen DN, Ellenbogen KA, Fitzpatrick AP, Scheinman MM. Tachycardia-induced cardiomyopathy: a review of animal models and clinical studies. J Am Coll Cardiology 1997; 29: 709-15.

4 Young $C R$, Bowers MB, Manure CM. Management of the adverse effects of clozapine. Schizophrenia Bull 1998; 24: 381-90.

William R. Jones is consultant psychiatrist, Yorkshire Centre for Eating Disorders, Leeds, Leeds and York Partnership NHS Foundation Trust, email: wrjones@doctors.org.uk, Usha Narayana is ST4 in psychiatry, Bootham Park Hospital, York, Leeds and York Partnership NHS Foundation Trust, Sarah Howarth is clinical audit officer, Joanna Shinners is clinical audit lead and Qadeer Nazar is consultant psychiatrist, all at Lynfield Mount Hospital, Bradford, Bradford District Care Trust.

doi: $10.1192 / p b .38 .3 .140 a$ 